\title{
6061-T6 铝合金/SPCC 冷轧钢激光熔钎焊特性研究*
}

\author{
张建勋 江 尤 徐甄真 张贵锋 \\ (西安交通大学金属材料强度国家重点实验室 西安 710049)
}

\begin{abstract}
摘要: 针对 $2 \mathrm{~mm}$ 厚的 6061-T6 铝合金与 SPCC 冷轧钢板, 采取填丝激光熔钎焊方法, 研究预涂钎剂、坡口角度、坡口形貌、 钎料成分对熔钎焊接头的组织成分及力学性能的影响。结果表明, 预涂 Nocolok 钎剂可以显著改善钎料的润湿性能; 在钢母 材侧预制 $60^{\circ}$ 坡口比预制 $45^{\circ}$ 坡口时的激光熔钎焊接头力学性能更好; 当对钢母材预制 $60^{\circ}$ 坡口时, 在激光功率、焊接速度、 送丝速度、离焦量、光斑中心的偏移距离分别为 $3.1 \mathrm{~kW} 、 0.5 \mathrm{~m} / \mathrm{min} 、 2 \mathrm{~m} / \mathrm{min} 、-8 \mathrm{~mm} 、+0.6 \mathrm{~mm}$ 时, 以 Al-Cu 焊丝作为填 充钎料, 界面形成短须状的 $\mathrm{Fe}-\mathrm{Al}$ 金属间化合物层, 厚度在 $5 \sim 8 \mu \mathrm{m}, \mathrm{Cu}$ 元素以置换 $\mathrm{Fe}$ 元素的方式固溶在 $\mathrm{Fe}-\mathrm{Al}$ 金属间化 合物相中改善其脆性, 接头的平均及最大抗拉强度分别为 $147 \mathrm{MPa} 、 159 \mathrm{MPa}$; 以 $\mathrm{Al}-\mathrm{Mg}$ 焊丝为填充钎料时, 界面形成锯齿 状的 $\mathrm{Fe}-\mathrm{Al}$ 金属间化合物层, 厚度在 $8 \sim 12 \mu \mathrm{m}, \mathrm{Mg}$ 元素仅聚集在脆性相外侧, 对 $\mathrm{Al}-\mathrm{Fe}$ 脆性相的生长无明显抑制作用, 在 激光功率为 $3 \mathrm{~kW}$ 时, 接头的平均抗拉强度为 $118 \mathrm{MPa}$; 以 $\mathrm{Al}-\mathrm{Si}$ 焊丝为填充钎料时, 界面形成小锯齿状的 Fe-Al 金属间化合 物层, 厚度在 3 4.5 $\mu \mathrm{m}, \mathrm{Si}$ 元素将固溶在 $\mathrm{Fe}-\mathrm{Al}$ 金属间化合物相中抑制其生长, 在激光功率为 $3 \mathrm{~kW}$ 时, 接头的平均抗拉强 度为 $129 \mathrm{MPa}$ 。
\end{abstract}

关键词: 铝钢接头; 激光熔钎焊; 预涂钎剂; 界面结构; 力学性能

中图分类号: TG456

\section{Study on Characteristics of Laser Welding-brazing of 6061 Aluminum Alloy to SPCC Cold Rolled Steel}

\section{ZHANG Jianxun JIANG Xu XU Zhenzhen ZHANG Guifeng}

(State Key Laboratory for Mechanical Behavior of Materials, Xi'an Jiaotong University, Xi'an 710049)

\begin{abstract}
T6 aluminum alloy and SPCC cold-rolled steel plate are used to obtain aluminum steel composite joints with both fusion and brazing characteristics by means of wire filling laser brazing method. The influences of pre-coated flux, groove bevel angle, bevel morphology and solder composition on the microstructure and mechanical properties of brazing joint are studied. The results show that pre-coating flux can greatly improve wettability of filler metal. The mechanical properties of laser brazing joint are better when $60^{\circ}$ groove is prefabricated on the side of steel base metal than when $45^{\circ}$ groove is prefabricated.. When the prefabricate groove angle of steel material is $60^{\circ}$, and the laser power, welding speed, wire feeding speed, laser defocusing amount, offset center of the laser spot center are $3.1 \mathrm{~kW}, 0.5 \mathrm{~m} / \mathrm{min}, 2 \mathrm{~m} / \mathrm{min},-8 \mathrm{~mm},+0.6 \mathrm{~mm}$, respectively, with ER2319 Al-Cu filler metal, the average and maximum tensile strength of the joint are respectively $147 \mathrm{MPa}$ and $159 \mathrm{MPa}$, the interface layer presents short whisker shape, the interface layer is 5-8 $\mu \mathrm{m}$ in thickness, the $\mathrm{Cu}$ element is dissolved in Al-Fe brittle phase by replacing Fe elements to reduce its brittleness. With ER5356 Al-Mg filler metal, the average tensile strength of the joint is $118 \mathrm{MPa}$ when the laser power is $3 \mathrm{~kW}$, the interface layer presents coarse saw tooth shape, and consists of $\mathrm{FeAl}_{2}, \mathrm{Fe}_{2} \mathrm{Al}_{5}$ and $\mathrm{FeAl}_{3}$ phase, the interface layer is 8-12 $\mu \mathrm{m}$ in thickness, the $\mathrm{Mg}$ element is gathered at the outside of brittle phase, and it has no obvious inhibitory effect on the growth of Al-Fe brittle phase; with ER4043 Al-Si filler metal, the average tensile strength of the joint is $129 \mathrm{MPa}$ when the laser power is $3 \mathrm{~kW}$, the interface layer presents small saw tooth shape, the interface layer is 3-4.5 $\mu \mathrm{m}$ in thickness, the Si element is dissolved in Al-Fe brittle phase to inhibit its growth.
\end{abstract}

Key words: aluminum-steel joint; laser welding-brazing; pre-coated flux; interface structure; mechanical property

* 国家自然科学基金资助项目(51875442)。20190809 收到初稿, 20191209 收到修改稿 


\section{0 前言}

随着中国经济快速发展, 机动车保有量与日俱 增, 由此引发的能源供给不足和环境污染问题也日 益严重。为减少对化石能源的消耗, 相关学者和研 究人员对混合动力、纯电动、燃料电池等新能源汽 车技术进行了研究 ${ }^{[1-2]}$, 汽车的燃料消耗和尾气排放 与汽车质量成正比, 车身重量每减轻 $100 \mathrm{~kg}$, 每百 公里油耗减少 $0.4 \mathrm{~L}, \mathrm{CO}_{2}$ 排放量减少 $1 \mathrm{~kg}$, 控制车 重可有效达到节能减排的目的。

异质材料焊接作为降低车重的关键技术之一正 被应用及进行专题研究 ${ }^{[3]}$, 其中, 铝/钢焊接备受关 注, 国内外诸多学者基于不同的焊接手段对铝/钢焊 接进行了大量研究。LI 等 ${ }^{[4]}$ 在平板爆炸焊的基础上 预先给钢板加工燕尾槽, 对 5083 铝合金和 Q345 钢 进行爆炸焊研究, 在铝合金与钢母材侧燕尾槽顶端、 底端界面处存在局部熔化的区域, 侧端界面表现为 连续的熔融层, 检测到 $\mathrm{FeAl}_{2} 、 \mathrm{Fe}_{2} \mathrm{Al}_{5}$ 脆性相, 试验 获得的接头剪切强度均在 $168 \mathrm{MPa}$ 以上。MACWAN 等 ${ }^{[5]}$ 对 6111-T4 铝合金与 HSLA 镀锌钢进行超声波 焊接, 在铝钢界面处生成 $\mathrm{Al}-\mathrm{Zn}$ 共晶组织, 当接头 预紧力、焊接能量分别为 $16.5 \mathrm{~N} 、 2000 \mathrm{~J}$ 时, 接头 最大抗剪切力为 $4.3 \mathrm{kN}$ 。LEITAO 等 ${ }^{[6]}$ 采用铝上钢下 的搭接方式对 6082-T6 铝合金与 S355 钢进行搭接摚 拌摩擦焊, 试验中搅拌头在铝母材上焊接三道, 接 头的最高强度达 $185 \mathrm{MPa}$, 此时钢母材侧的热机影 响区厚约 $5 \mu \mathrm{m}$, 在铝钢界面处未发现脆性相组织, 接头主要靠波浪状界面和元素的扩散/固溶作用实 现有效连接。LI 等 ${ }^{[7]}$ 在 TIG 熔钎焊的基础上添加激 光热源对 5A06 铝合金和 ST04Z 镀锌钢进行焊接, 接头最大强度为 $151 \mathrm{MPa}$ 。王晓虹等 ${ }^{[8]}$ 采用钢上铝 下的搭接方式, 对 6082 铝合金和 $05 \mathrm{CuPCrNi}$ 耐候 钢进行激光焊接, 当添加 $\mathrm{Fe}-\mathrm{B}-\mathrm{Si}$ 中间层时, 接头 的抗剪力是不添加中间层的 1.37 倍, 此时铝/钢界面 更加平整, 岛状区域数量减少。另有免用钎剂、宽 焊道的搅拌摩擦钎焊报道 ${ }^{[9]}$ 。

由于激光填丝熔钎焊兼具激光能量及作用位置 可精确控制, 填充焊丝可准确调控填充金属成分配 比的优势，本文采用预制坡口的平板对接的方式， 对 $2 \mathrm{~mm}$ 厚的 6061-T6 铝合金与 SPCC 冷轧钢板进 行了预涂钎剂填丝激光熔钎焊, 避免了搭接方式导 致的板材浪费问题，获得了良好的铝/钢焊接接头, 并研究了对接坡口角度、坡口形貌与钎料成分对熔 钎焊接头的组织成分与力学性能的影响。

\section{1 试验材料与方法}

\section{1 试验材料}

试验母材选用 6061-T6 铝合金和 SPCC 冷轧钢, 冷轧钢板表面未做热镀锌处理。母材试板的尺寸均 为 $2 \mathrm{~mm} \times 60 \mathrm{~mm} \times 120 \mathrm{~mm}$, 其主要化学成分与力学 性能如表 $1 、 2$ 所示。

表 1 母材的化学成分(质量分数)

\begin{tabular}{ccccccccc}
\hline 元素 & $\mathrm{Al}$ & $\mathrm{Si}$ & $\mathrm{Fe}$ & $\mathrm{C}$ & $\mathrm{Mn}$ & $\mathrm{Mg}$ & $\mathrm{Cr}$ & $\mathrm{Zn}$ \\
\hline $6061-\mathrm{T} 6$ & 余量 & 0.52 & 0.25 & - & 0.012 & 0.95 & 0.26 & 0.02 \\
$\mathrm{SPCC}$ & - & 0.30 & 余量 & 0.15 & 0.60 & - & - & - \\
\hline
\end{tabular}

表 2 母材的力学性能

\begin{tabular}{cccc}
\hline 力学性能 & 屈服强度/MPa & 抗拉强度 $/ \mathrm{MPa}$ & 伸长率 $(\%)$ \\
\hline $6061-\mathrm{T} 6$ & $\geqslant 240$ & $\geqslant 290$ & $\geqslant 9$ \\
$\mathrm{SPCC}$ & $130 \sim 260$ & $\geqslant 270$ & $\geqslant 28$ \\
\hline
\end{tabular}

填充钎料, 分别选择添加 $\mathrm{Mg} 、 \mathrm{Si} 、 \mathrm{Cu}$ 元素的 铝基焊丝，其对应的牌号分别为 ER5356 铝镁焊 丝、ER4043 铝硅焊丝、ER2319 铝铜焊丝, 焊丝 直径均为 $1.2 \mathrm{~mm}$, 化学成分及物理性能如表 $3 、 4$ 所示。

表 3 铝基焊丝的化学成分(质量分数) \%

\begin{tabular}{ccccccc}
\hline 元素 & $\mathrm{Al}$ & $\mathrm{Mg}$ & $\mathrm{Si}$ & $\mathrm{Cu}$ & $\mathrm{Fe}$ & $\mathrm{Mn}$ \\
\hline ER5356 & 余量 & $4.5 \sim 5.5$ & $<0.25$ & $<0.1$ & $<0.4$ & $0.05 \sim 0.2$ \\
ER4043 & 余量 & $<0.05$ & $4.5 \sim 6.0$ & $<0.3$ & $<0.6$ & $<0.05$ \\
ER2319 & 余量 & $<0.02$ & $<0.2$ & $5.5 \sim 6.8$ & $<0.3$ & $0.2 \sim 0.4$ \\
\hline
\end{tabular}

表 4 铝基焊丝的物理性能

\begin{tabular}{cccccc}
\hline 性能 & $\begin{array}{c}\text { 屈服强度/ } \\
\mathrm{MPa}\end{array}$ & $\begin{array}{c}\text { 抗拉强度/ } \\
\mathrm{MPa}\end{array}$ & $\begin{array}{c}\text { 伸长率 } \\
(\%)\end{array}$ & $\begin{array}{c}\text { 熔点/ } \\
{ }^{\circ} \mathrm{C}\end{array}$ & $\begin{array}{c}\text { 密度/ } \\
\left(\mathrm{g} \cdot \mathrm{cm}^{-3}\right)\end{array}$ \\
\hline ER5356 & $110 \sim 120$ & $240 \sim 296$ & $17 \sim 26$ & $562 \sim 633$ & 2.64 \\
ER4043 & $20 \sim 40$ & $120 \sim 165$ & $3 \sim 18$ & $573 \sim 625$ & 2.68 \\
ER2319 & $160 \sim 180$ & $240 \sim 270$ & 3 & $543 \sim 643$ & 2.77 \\
\hline
\end{tabular}

钎剂选用成分为 $\mathrm{K}_{1-3} \mathrm{AlF}_{3-6} / \mathrm{Li}_{3} \mathrm{AlF}_{6}$ 的 Nocolok 氟化物钎剂, 以改善钎料润湿性, 其化学成分及物 理性能如表 5、 $6^{[10-12]}$ 所示。

表 5 Nocolok 钎剂的化学成分(质量分数) \%

\begin{tabular}{|c|c|c|c|c|c|c|}
\hline 元素 & F & $\mathrm{K}$ & $\mathrm{Al}$ & $\mathrm{Li}$ & $\mathrm{Fe}$ & $\mathrm{Ca}$ \\
\hline \multirow[t]{2}{*}{ Nocolok } & $50 \sim 54$ & $26 \sim 30$ & $16 \sim 18$ & $\geqslant 0.5$ & $\leqslant 0.03$ & $\leqslant 0.1$ \\
\hline & 表 6 & \multicolumn{5}{|c|}{ Nocolok 钎剂的物理性能 } \\
\hline 性能 & $\begin{array}{c}\text { 密度/ } \\
\left(\mathrm{g} \cdot \mathrm{cm}^{-3}\right)\end{array}$ & \multicolumn{2}{|c|}{$\begin{array}{c}\text { 体密度/ } \\
\left(\mathrm{g} \cdot \mathrm{L}^{-1}\right)\end{array}$} & $\begin{array}{c}\text { 熔点/ } \\
{ }^{\circ} \mathrm{C}\end{array}$ & \multicolumn{2}{|c|}{$\begin{array}{c}\text { 水溶解度/ } \\
\left(\mathrm{g} \cdot \mathrm{L}^{-1}\right)\end{array}$} \\
\hline Nocolok & $2.8 \sim 3.0$ & \multicolumn{2}{|c|}{$350 \sim 550$} & $569 \sim 577$ & \multicolumn{2}{|c|}{$\approx 4.5$} \\
\hline
\end{tabular}




\section{2 激光熔钎焊}

激光熔钎焊系统由五个部分组成, 分别为光纤 激光源、激光头、机器人系统、送丝系统、气体保 护装置, 在试验过程中通过机器人控制器调整焊接 路径及焊接参数。激光熔钎焊试验装置实物与原理 见图 1, 夹具及气体保护装置实物见图 1a, 各装置 相对位置原理见图 1b。

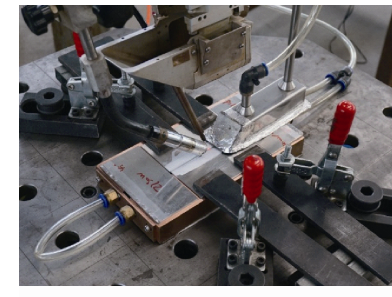

(a) 实物图

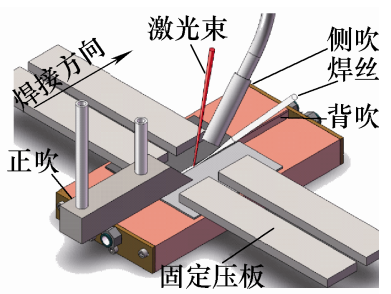

(b) 原理图
图 1 激光熔钎焊装置图

由于铝合金母材极易氧化, 试验前使用铣刀去 除对接面处的毛边及氧化膜, 浸入无水乙醇中超声 清洗 $1 \mathrm{~min}$, 再依次浸入质量分数 $10 \%$ 的 $\mathrm{NaOH}$ 溶 液、 $15 \%$ 的 $\mathrm{HNO}_{3}$ 溶液各 $1 \mathrm{~min}$, 最后使用无水乙醇 清洗, 烘干后存放于干燥箱中; 对钢母材在其对接 端面加工出不同角度、形貌的坡口, 用砂纸打磨后 置于无水乙醇中超声清洗 $1 \mathrm{~min}$, 烘干存放。

焊前将 Nocolok 氟化物钎剂倒入乙醇中配置质 量分数为 $25 \%$ 的钎剂溶液, 用毛刷蘸取后均匀涂覆 在铝/钢母材的对接端面上, 以能覆盖母材上下表面 距界面 $20 \mathrm{~mm}$ 宽的区域为宜, 待乙醇完全挥发后再 进行激光熔钎焊。

\section{3 微观组织与性能测试}

对于铝/钢异种金属熔化焊, 固/液界面是以 $\mathrm{Fe}$ 和 $\mathrm{Al}$ 的反应扩散生成金属间化合物。大量研究表 明，在界面可能形成五种 Fe-Al 金属间化合物，如 $\mathrm{Fe}_{3} \mathrm{Al} 、 \mathrm{FeAl} 、 \mathrm{FeAl}_{2} 、 \mathrm{Fe}_{2} \mathrm{Al}_{5}$ 和 $\mathrm{FeAl}_{3}$, 其中又以 $\mathrm{Fe}_{2} \mathrm{Al}_{5} 、 \mathrm{FeAl}_{3}$ 为最常见成分 ${ }^{[13-16]}$ 。通过 $\mathrm{EDS}$ 测试元 素成分含量, 虽不能完全确定属于何种金属间化合 物, 但可结合 Fe-Al 合金相图, 定性推断 Fe-Al 金 属间化合物的大致种类 ${ }^{[17-18]}$ 。

金相组织及成分分析使用 Keller 试剂, 其成分 配比为 $1 \mathrm{~mL} \mathrm{HF}+1.5 \mathrm{~mL} \mathrm{HCl}+2.5 \mathrm{~mL} \mathrm{HNO}_{3}+95 \mathrm{~mL}$ $\mathrm{H}_{2} \mathrm{O}$, 腐蚀时间控制在 5 10 s。显微组织观察及化 学元素检测, 均在 Merlin Compact 场发射扫描电镜 上进行。为评价焊接接头的强度, 沿着焊接方向等 间隔切取 3 个拉伸试样，其尺寸依据金属材料室温 拉伸国家标准 GB228-2002 制备, 将焊接后试样上 下表面的余高铣除并打磨后, 使用万能试验机, 进
行静态拉伸试验, 引伸计标距为 $53.5 \mathrm{~mm}$, 拉伸速 率固定在 $0.5 \mathrm{~mm} / \mathrm{min}$ 。

\section{2 试验结果与讨论}

\section{1 预涂钎剂的作用}

为研究预涂钎剂在激光熔钎焊中的作用, 基于 图 2 所示的原理与方法进行预涂钎剂对钢材的润湿 性对比试验。

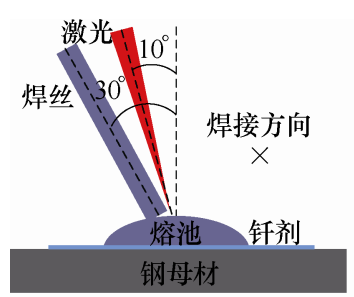

(a) 原理

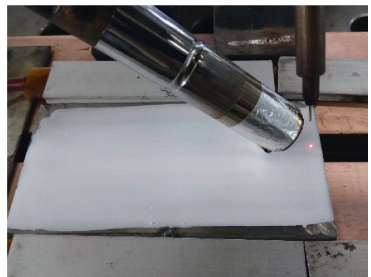

(b) 试验
图 2 激光加热下润湿试验

焊接参数保持不变, 激光功率、焊接速度分别 为 $2000 \mathrm{~W} 、 0.5 \mathrm{~m} / \mathrm{min}$, 激光束与垂直面成 $10^{\circ}$ 夹角, 离焦量为 $-8 \mathrm{~mm}$, 送丝方向与垂直面成 $30^{\circ}$ 夹角, 送 丝速度 $2.35 \mathrm{~m} / \mathrm{min}$, 侧吹保护气体流量为 $20 \mathrm{~L} / \mathrm{min}^{\circ}$ 。

对于填充 ER4043 铝硅焊丝钎料, 在试验完成 后沿垂直焊接方向截取金相试样。无预涂钎剂时的 试样截面图见图 3a, 熔池在钢母材表面润湿极差, 钎料收缩为球状。预涂钎剂时的试样截面见图 3b, 熔池在钢母材铺展, 润湿性良好。

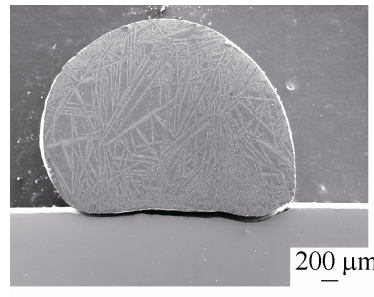

(a) 无预涂钎剂

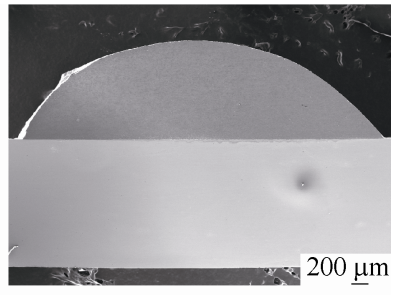

(b) 预涂钎剂
图 3 预涂钎剂对铝硅钎料润湿的影响

为定量表征预涂钎剂对钎料润湿性的改善效 果, 以熔化钎料在钢母材表面的平均润湿角为参考。 对于 ER4043 铝硅焊丝钎料, 预涂钎剂后, 钎料在 钢材表面的平均润湿角由 $124^{\circ}$ 降低至 $75^{\circ}$ 。对于 ER5356 铝镁焊丝钎料和 ER2319 铝铜焊丝钎料, 其 各自平均润湿角分别由 $117^{\circ}$ 降低至 $87^{\circ}$ 、由 $134^{\circ}$ 降低至 $74^{\circ}$ 。可见预涂钎剂对三种焊丝钎料在钢材 表面的润湿铺展过程均有改善作用。

采用能谱仪 (Energy dispersive spectrometer, EDS)检测填充铝硅钎料时, 激光熔钎焊试样各区域 的化学元素含量, 无预涂钎剂时, 钎缝与钢母材界 面及其内部的组织及元素测点结果见图 4、表 7。 


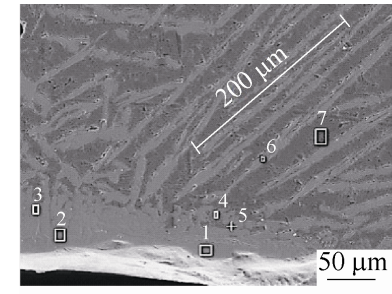

(a) 界面 (b) 钎缝

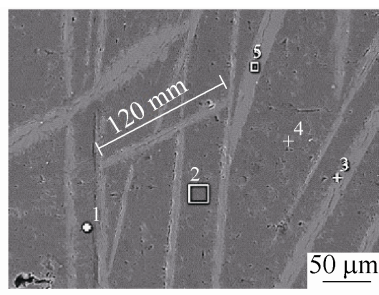

图 4 无预涂钎剂的界面与钎缝 EDS 测点

表 7 无预涂钎剂的界面与钎缝 EDS 分析(原子分数) \%

\begin{tabular}{ccccccc}
\hline & 测点 & $\mathrm{Al}$ & $\mathrm{Mg}$ & $\mathrm{Si}$ & $\mathrm{Cu}$ & $\mathrm{Fe}$ \\
\hline & 1 & 65.06 & 0.09 & 3.51 & 0.14 & 31.21 \\
& 2 & 67.72 & 0.10 & 2.97 & 0.13 & 29.09 \\
界 & 3 & 69.39 & 0.09 & 2.56 & 0.09 & 27.87 \\
面 & 4 & 72.25 & 0.00 & 2.57 & 0.09 & 25.08 \\
& 5 & 98.40 & 0.00 & 0.72 & 0.00 & 0.88 \\
& 6 & 71.70 & 0.11 & 1.95 & 0.04 & 26.20 \\
& 7 & 94.55 & 0.01 & 4.31 & 0.00 & 1.12 \\
\hline & 1 & 93.17 & 0.04 & 0.58 & 0.00 & 6.21 \\
& 2 & 89.98 & 0.11 & 9.22 & 0.00 & 0.69 \\
钎 & 3 & 75.28 & 0.07 & 8.82 & 0.12 & 15.70 \\
& 4 & 99.12 & 0.00 & 0.62 & 0.00 & 0.27 \\
& 5 & 71.74 & 0.04 & 1.71 & 0.04 & 26.46 \\
\hline
\end{tabular}

在亮色区域的 $\mathrm{Fe}$ 元素比例高, 暗色区域的 $\mathrm{Fe}$ 元素含量低。由图 4a 可见, 钎缝与钢母材界面存在 明显缺口, 钎缝中的 $\mathrm{Fe}$ 元素应是来源于钢母材表面 的局部熔化, 熔化的 $\mathrm{Fe}$ 元素与 $\mathrm{Al}$ 结合形成大量长 度在 $100 \sim 200 \mu \mathrm{m}$, 交错排布的针状 $\mathrm{Fe}-\mathrm{Al}$ 金属间 化合物, 在针状组织的边界, 即图 $4 b$ 的 3 号检测点, 分析得 $\mathrm{Al} 、 \mathrm{Fe} 、 \mathrm{Si}$ 的原子比约为 $8.5: 1.8: 1$, 其 可能是 $\tau_{5}-\mathrm{Al}_{8} \mathrm{Fe}_{2} \mathrm{Si}$ 相 ${ }^{[19]}$ 。

预涂钎剂情况下的结果见图 5、表 8, 可见 $\mathrm{Fe}$ 元素集中在界面附近, 形成 Fe-Al 金属间化合物, 厚度为 $50 \sim 60 \mu \mathrm{m}$, 钎缝内部主要是 $\alpha-\mathrm{Al}$ 固溶体和 $\mathrm{Al}-\mathrm{Si}$ 共晶组织。观察预涂钎剂引入的 $\mathrm{F}$ 元素在界面 的分布规律, 发现其含量沿钢母材向钎缝方向先增 后降, 在界面过渡层中, 即图 $5 \mathrm{a}$ 的 3 号检测点 $\mathrm{F}$ 元素的原子百分比达到 $1.08 \%$, 推测焊接过程中熔 池快速冷却, 部分钎剂残留在界面处。对比可见, 预涂钎剂不仅改善了熔池在钢母材表面的润湿性 能, 还有效降低了焊接过程中热量向钢母材的传递,

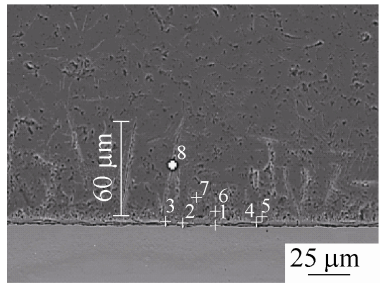

(a) 界面

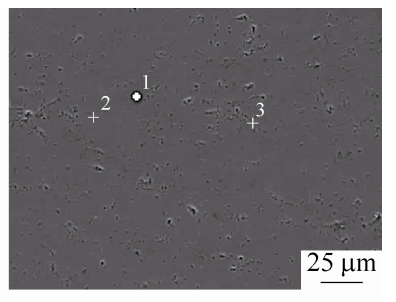

(b) 钎缝
图 5 预涂钎剂的界面与钎缝 EDS 测点
表 8 预涂钎剂界面与钎缝 EDS 分析(原子分数) \%

\begin{tabular}{|c|c|c|c|c|c|c|c|}
\hline & 测点 & $\mathrm{Al}$ & $\mathrm{Mg}$ & $\mathrm{Si}$ & $\mathrm{Cu}$ & $\mathrm{Fe}$ & $\mathrm{F}$ \\
\hline \multirow{8}{*}{ 界面 } & 1 & 38.17 & 0.05 & 3.59 & 0.19 & 57.39 & 0.62 \\
\hline & 2 & 55.69 & 0.09 & 9.03 & 0.01 & 34.17 & 1.01 \\
\hline & 3 & 68.19 & 0.03 & 3.22 & 0.13 & 27.35 & 1.08 \\
\hline & 4 & 66.65 & 0.13 & 3.61 & 0.09 & 28.66 & 0.85 \\
\hline & 5 & 80.65 & 0.00 & 5.13 & 0.05 & 13.59 & 0.57 \\
\hline & 6 & 91.10 & 0.00 & 3.91 & 0.06 & 4.45 & 0.48 \\
\hline & 7 & 96.72 & 0.01 & 1.90 & 0.02 & 1.08 & 0.27 \\
\hline & 8 & 75.54 & 0.09 & 9.42 & 0.03 & 14.34 & 0.57 \\
\hline \multirow{3}{*}{ 钎缝 } & 1 & 95.70 & 0.10 & 3.78 & 0.00 & 0.03 & 0.40 \\
\hline & 2 & 98.50 & 0.06 & 1.33 & 0.02 & 0.00 & 0.10 \\
\hline & 3 & 96.32 & 0.01 & 3.48 & 0.00 & 0.03 & 0.16 \\
\hline
\end{tabular}

减弱了界面钢母材的熔化过程, 钎剂层起到了热量 壁垒、化学壁垒的作用。

\section{2 坡口角度对接头强度的影响}

为研究坡口角度对焊接接头力学性能的影响, 分别对钢母材预制了 $45^{\circ}$ 和 $60^{\circ}$ 两种坡口, 预涂钎剂 后焊接, 填充钎料为铝硅焊丝。焊接示意图及取样 示意图见图 6。

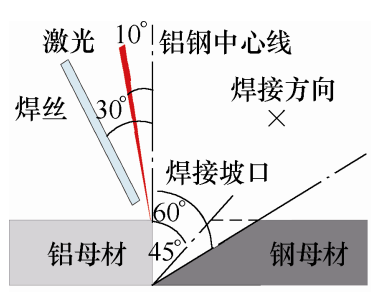

(a) 焊接示意图

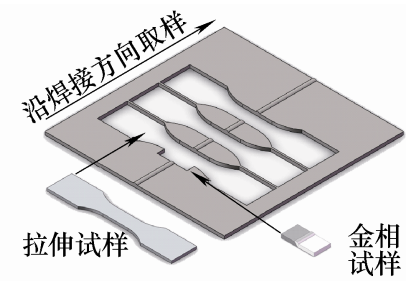

(b) 取样示意图
图 6 铝钢激光熔钎焊示意图

为避免钢母材的过多熔化, 激光光斑以铝/钢界 面为中心向铝侧偏移 $+0.6 \mathrm{~mm}$; 焊丝顶端到铝母材 的距离为 $10 \mathrm{~mm}$, 激光先熔化铝材再熔化焊丝, 焊 丝干伸长固定为 $5 \mathrm{~mm}$, 送丝方向与垂直面夹角为 $30^{\circ}$, 激光光束的入射方向与垂直面夹角为 $10^{\circ}$, 选 择纯氩气作为保护气体, 侧吹、正吹、背吹三个方 向的气体流量均设置为 $20 \mathrm{~L} / \mathrm{min}$ 。激光的离焦量及 光斑直径分别为 $-8 \mathrm{~mm} 、 1.2 \mathrm{~mm}$, 激光焊接速度与 送丝速度分别为 $0.5 \mathrm{~m} / \mathrm{min} 、 2 \mathrm{~m} / \mathrm{min}$ 。

在激光熔钎焊中, 送丝速度对钎缝成形具有直 接影响。送丝速度快, 会使激光熔钎焊的稳定性变 差; 送丝速度慢, 会导致钎缝成形的不连续, 产生 成形缺陷。通常是基于需要填充的钎缝体积, 按照 式(1)选择合适的送丝速度

$$
k \times S \times v_{f}=\frac{\pi}{4} \times d^{2} \times v_{s}
$$

式中, $k$ 为钎缝成形系数; $S$ 为钢母材预制坡口后需 填充区域截面的直角三角形面积 $\left(\mathrm{m}^{2}\right) ; v_{f}$ 为焊接速 度 $\left(\mathrm{m} \cdot \mathrm{min}^{-1}\right) ; d$ 为填充焊丝直径 $(\mathrm{m}) ; v_{s}$ 为送丝速度 
$\left(\mathrm{m} \cdot \mathrm{min}^{-1}\right)$ 。

不同坡口角度的焊接接头横截面形貌见图 7, 其中图 7a、7c、7e、7 $、 7 \mathrm{i}$ 是坡口角度为 $45^{\circ}$ 的试 样，图 7b、7d、7f、7h、7j 是坡口角度为 $60^{\circ}$ 的试 样, 由于坡口角度越大需填充的焊缝体积越大, 所 需激光能量也应增加, 故 $60^{\circ}$ 坡口角度的试样, 其 激光功率在 $45^{\circ}$ 坡口角度试样的基础上增加 $10 \%$ 。

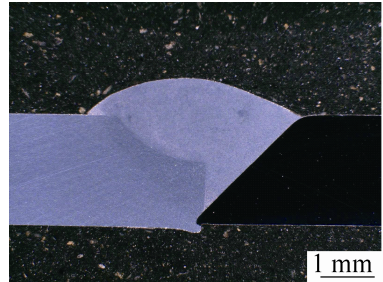

(a) $2 \mathrm{~kW}, 45^{\circ}$

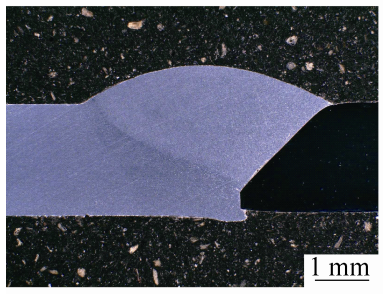

(c) $2.25 \mathrm{~kW}, 45^{\circ}$

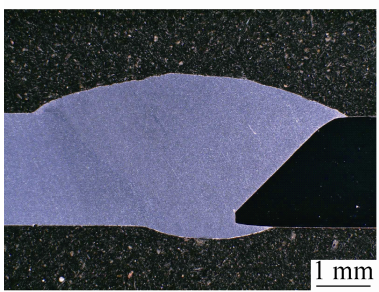

(e) $2.5 \mathrm{~kW}, 45^{\circ}$

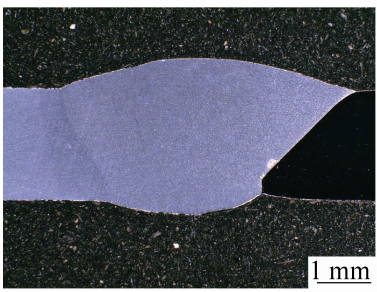

(g) $2.75 \mathrm{~kW}, 45^{\circ}$

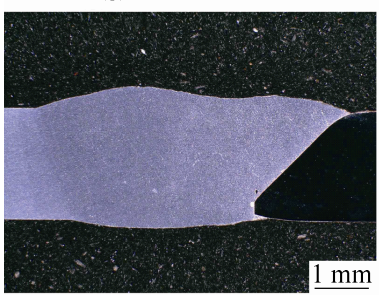

(i) $3 \mathrm{~kW}, 45^{\circ}$

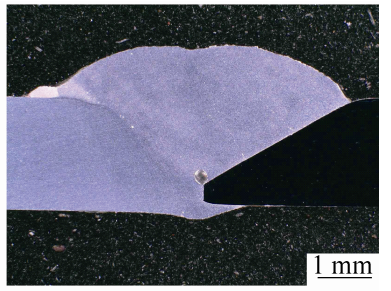

(b) $2.2 \mathrm{~kW}, 60^{\circ}$

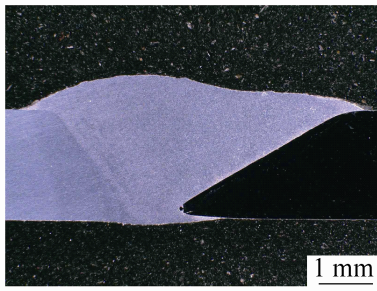

(d) $2.475 \mathrm{~kW}, 60^{\circ}$

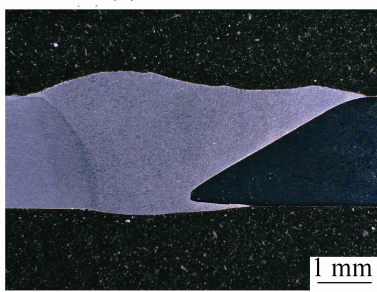

(f) $2.75 \mathrm{~kW}, 60^{\circ}$

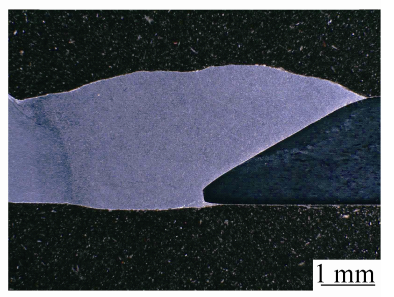

(h) $3.025 \mathrm{~kW}, 60^{\circ}$

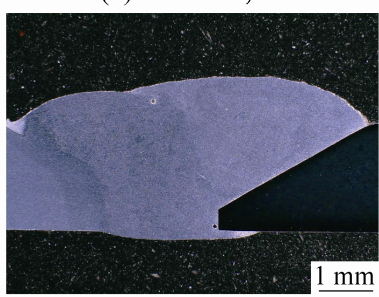

(j) $3.3 \mathrm{~kW}, 60^{\circ}$
图 7 不同坡口角度激光熔钎焊接头横截面

试验结果表明, 当预制坡口角度为 $45^{\circ}$, 激光 功率为 $2 \mathrm{~kW} 、 2.25 \mathrm{~kW}$ 时, 焊缝底部仍有较多的固 态铝母材, 从 $2.5 \mathrm{~kW}$ 的功率开始, 焊缝底部的固态 铝母材完全消失, 焊接接头完全焊透。当预制坡口 角度为 $60^{\circ}$, 激光功率为 $2.2 \mathrm{~kW}$ 时, 试样在焊缝底 部存在气孔缺陷, 当激光功率为 $2.475 \mathrm{~kW} 、 2.75 \mathrm{~kW}$ 时焊丝尚可完全填充母材的预留间隙, 但当功率进
一步增大至 $3.3 \mathrm{~kW}$ 时, 在铝母材的上表面处存在部 分咬边的现象。

沿垂直焊接方向对每组试样切取 3 个标准拉伸 试样，铣除表面余高后打磨平整，不同参数下接头 抗拉强度见图 8, 此处基于算术平均值及极值与平 均值的差值绘制上下不等的误差棒图，可见在同一 参数下接头的抗拉强度相差值最大可达 $30 \mathrm{MPa}$, 这 主要是由于焊接过程中, 不同取样区域的温度场存 在较大差异的缘故。

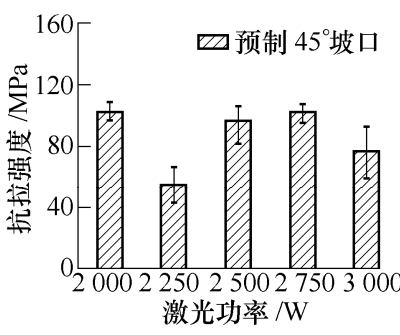

(a) $45^{\circ}$ 坡口

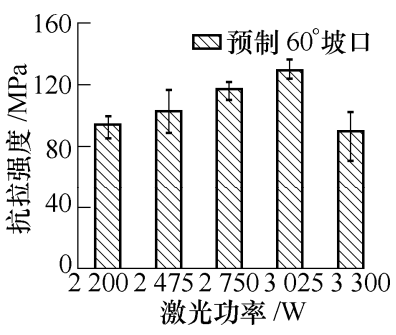

(b) $60^{\circ}$ 坡口
图 8 不同坡口角度接头抗拉强度

对于 $45^{\circ}$ 坡口的试样, 试样的平均抗拉强度在 $80 \sim 100 \mathrm{MPa}$ 间上下浮动, 均断裂在钎缝与钢母材 界面, 当激光功率为 $2.25 \mathrm{~kW}$ 时, 由于界面下侧钎 缝与钢母材未完全熔合, 界面处存在部分间隙, 导 致接头力学性能急剧下降, 当激光功率为 $2.75 \mathrm{~kW}$ 时, 接头平均抗拉强度达 $102 \mathrm{MPa}$ 。对于 $60^{\circ}$ 坡口试 样, 随激光功率增大, 试样的平均抗拉强度先增后 减, 在激光功率为 $3.025 \mathrm{~kW}$ 时, 试样的平均抗拉强 度达 $129 \mathrm{MPa}$ 。

通过对另外两种焊丝的强度试验结果发现, $60^{\circ}$ 坡口的试样的抗拉强度均大于 $45^{\circ}$ 坡口的试样, 断 裂位置均在钎缝与钢母材界面, 可以推断在同样焊 接材料情况下, 钎缝与钢母材的界面结合状态基本 相同。因此接头强度增加是由于坡口角度增大, 导 致界面面积增加所致。

\section{3 坡口开 $\mathrm{V}$ 型槽对接头强度的影响}

通过坡口角度的对比试验, 可知坡口增大可 提高接头力学性能, 同时在测量时发现试样在获 得最大抗拉强度后, 均表现为先在钎缝与钢母材的 下侧界面处起裂, 而后在上侧界面处断裂, 故在钢 母材的下表面处预制 2 道 $\mathrm{V}$ 型槽, 如图 9 所示, 意

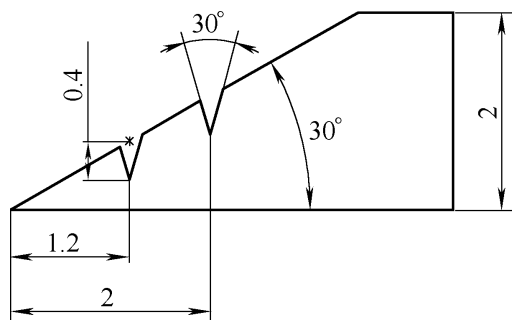

图 9 开 $\mathrm{V}$ 型槽的尺寸及位置 
图通过增大接头下侧铝与钢的接触面积, 起到 “钩 爪” 效果, 改善接头的力学性能, 激光试验参数见 表 9。

表 9 试验参数选择

\begin{tabular}{cccccc}
\hline 方案 & $\begin{array}{c}\text { 处理 } \\
\text { 方式 }\end{array}$ & $\begin{array}{c}\text { 激光功率/ } \\
\mathrm{kW}\end{array}$ & $\begin{array}{c}\text { 焊接速度/ } \\
\left(\mathrm{m} \cdot \mathrm{min}^{-1}\right)\end{array}$ & $\begin{array}{c}\text { 送丝速度/ } \\
\left(\mathrm{m} \cdot \mathrm{min}^{-1}\right)\end{array}$ & $\begin{array}{c}\text { 离焦量/ } \\
\mathrm{mm}\end{array}$ \\
\hline 1 & 无 & 3.025 & 0.5 & 2.00 & -8 \\
2 & 开槽 & 3.075 & 0.5 & 2.05 & -8 \\
\hline
\end{tabular}

两种试验方案条件下接头横截面及断口侧面 宏观形貌见图 10。可见对于方案 2, 铝硅焊丝的 流动性较好, 能有效填充预制的 $\mathrm{V}$ 型槽, 同时对 断口侧面的观察可见, 方案 1 的断口基本沿着焊 缝与钢母材的界面断裂, 而方案 2 中除了在界面 处断裂外, 从焊缝延伸到钢母材 $\mathrm{V}$ 型槽的部分也 发生了断裂, 焊缝的延伸部分在试样拉伸断裂后 还残留在钢母材上, $\mathrm{V}$ 型槽的存在似乎发挥了 “钩 爪”的效果。

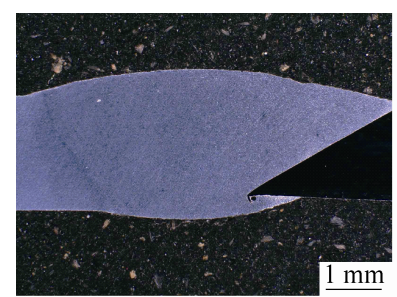

(a) 方案 1 横截面

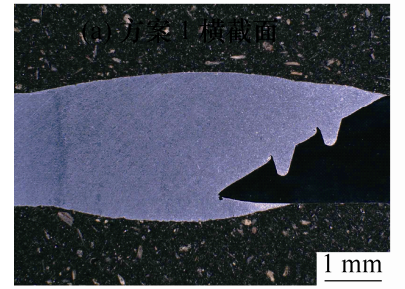

(c) 方案 2 横截面

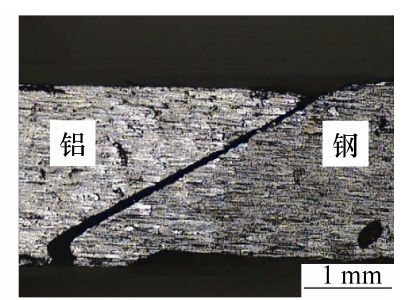

(b) 方案 1 断口截面

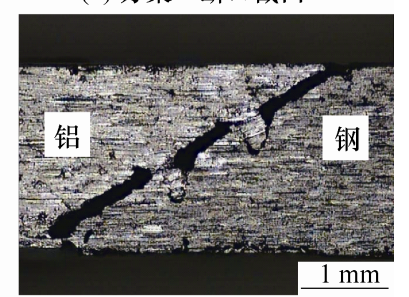

(d) 方案 2 断口截面
图 10 不同坡口形貌方案接头截面

两种方案下试样的焊接接头力学性能测试结果 见图 11, 从试样应力应变曲线可见, 应力在达到最 大值后, 均表现为先迅速下降而后逐渐上升, 最后 再逐渐下降。观察试样的拉伸过程, 发现方案 1 出 现这种现象是由于接头的下侧先发生断裂, 此时上 侧保持连接的状态, 但伴随裂纹的扩展, 接头最终 完全断裂; 方案 2 则是在上述机理的基础上, 还附 加有 $\mathrm{V}$ 型槽 “钩爪” 的作用。但是从抗拉强度的对 比结果还是发现, 方案 2 的性能不及方案 1 , 这可 能是由于 $\mathrm{V}$ 型槽将钢与焊缝的接触面分成多个独立 区域, 导致有效接触面积不增反降, 且界面形貌的 改变, 导致界面处在拉伸时应力集中严重, 对接头 力学性能不利。

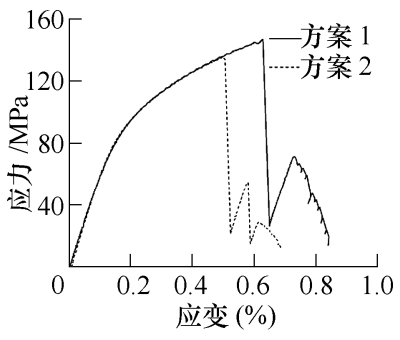

(a) 应力应变曲线

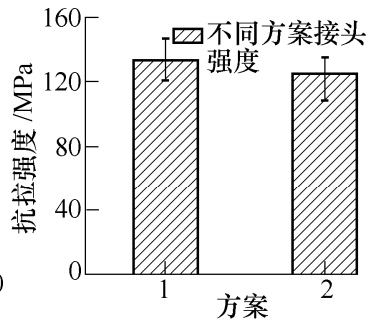

(b) 抗拉强度
图 11 不同坡口形貌接头的力学性能

拉伸试样断口与 EDS 元素分析结果见图 12、 表 10。由图 12a 可见, 方案 1 试样的上侧, 即元 素测点 1 和 2 处的含 $\mathrm{Fe}$ 量低, 此处主要组织为 $\alpha-\mathrm{Al}$ 固溶体, 表明其断裂在钎缝, 试样的下侧, 即元素 测点 3 5 处的含 $\mathrm{Fe}$ 量高, 主要组织为 $\mathrm{Fe}-\mathrm{Al}$ 金属 间化合物, 表明其断裂在界面。对于图 $12 \mathrm{~b}$ 所示 的方案 2 , 试样在 $V$ 型槽处残留组织主要为 $\alpha-A 1$ 固溶体, 而在平齐断口处, 主要是 $\mathrm{Fe}-\mathrm{Al}$ 金属间化 合物。

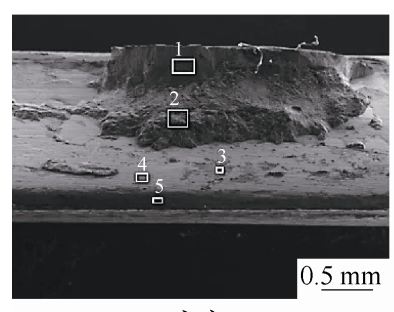

(a) 方案 1

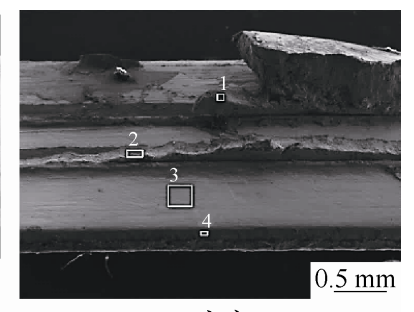

(b) 方案 2
图 12 不同坡口接头拉伸断口形貌

表 10 钢材侧断口 EDS 分析(原子分数) \%

\begin{tabular}{ccccccc}
\hline & 测点 & $\mathrm{Al}$ & $\mathrm{Mg}$ & $\mathrm{Si}$ & $\mathrm{Cu}$ & $\mathrm{Fe}$ \\
\hline & 1 & 96.14 & 0.66 & 2.67 & 0.06 & 0.48 \\
方 & 2 & 93.18 & 0.94 & 4.91 & 0.11 & 0.87 \\
案 & 3 & 72.91 & 2.23 & 2.37 & 0.01 & 22.49 \\
1 & 4 & 55.52 & 0.38 & 3.13 & 0.13 & 40.84 \\
& 5 & 81.84 & 0.83 & 2.17 & 0.02 & 15.15 \\
\hline \multirow{2}{*}{ 方 } & 1 & 90.97 & 0.71 & 5.64 & 0.09 & 2.59 \\
案 & 2 & 93.37 & 0.60 & 4.95 & 0.11 & 0.97 \\
2 & 3 & 49.94 & 0.17 & 3.87 & 0.06 & 45.96 \\
& 4 & 66.32 & 0.77 & 3.30 & 0.08 & 29.52 \\
\hline
\end{tabular}

\section{4 填充焊丝对接头强度的影响}

为分析不同合金元素对熔钎焊接头性能的影 响, 进行不同合金成分焊丝的对比试验。焊接功率 分别为 $3 \mathrm{~kW}, 3.1 \mathrm{~kW}, 3.2 \mathrm{~kW}$; 其他焊接参数与第 2.2 节相同。焊接接头力学性能测试结果见图 13, 可见激光功率对抗拉强度有一定的影响, 填充 $\mathrm{Al}-\mathrm{Cu}$ 焊丝的焊接接头, 其力学性能在焊接功率为 $3.1 \mathrm{~kW}$ 时最佳, 此时平均及最大抗拉强度分别达到 $147 \mathrm{MPa} 、 159 \mathrm{MPa}$, 填充其他两种焊丝时, 焊接接 头的抗拉强度随激光功率增加有所下降。 


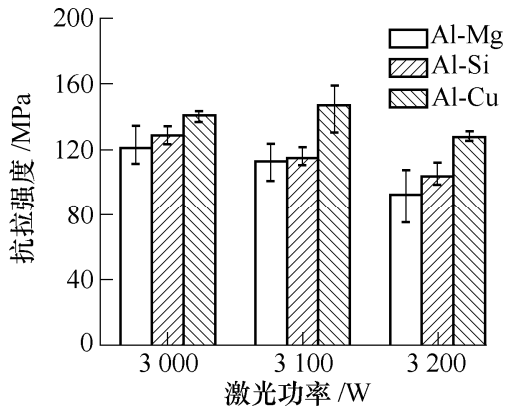

图 13 不同焊丝下接头的力学性能

填充不同焊丝时, 力学性能最佳试样所对应的激 光功率、界面过渡层厚和达到铝母材的强度比见表 11, 结果表明填充 $\mathrm{Al}-\mathrm{Cu}$ 焊丝时, 接头的强度比最高, 达到 $50 \%$, 填充 $\mathrm{Al}-\mathrm{Si}$ 焊丝时强度比为 $45 \%$, 填充 $\mathrm{Al}-\mathrm{Mg}$ 焊丝时强度比最低, 仅为 $40 \%$ 。对比三种焊接 接头的界面层厚度, 发现 $\mathrm{Al}-\mathrm{Si}$ 最薄, $\mathrm{Al}-\mathrm{Cu}$ 次之, $\mathrm{Al}-\mathrm{Mg}$ 最厚。研究表明, 对于铝/钢异种金属焊接接头, 其界面化合物层厚需控制在 $10 \mu \mathrm{m}$ 以下, 过厚的化合 物易导致自身脆裂，过薄则易造成结合不良 ${ }^{[17]}$ 。

表 11 典型试样的接头性能

\begin{tabular}{ccccc}
\hline 焊丝成分 & 激光功率 $/ \mathrm{kW}$ & 抗拉强度 $/ \mathrm{MPa}$ & 界面层厚度/ $\mu \mathrm{m}$ & 强度比 $(\%)$ \\
\hline $\mathrm{Al}-\mathrm{Mg}$ & 3.0 & 118 & $8 \sim 12$ & 40 \\
$\mathrm{Al}-\mathrm{Si}$ & 3.0 & 129 & $3 \sim 4.5$ & 45 \\
$\mathrm{Al}-\mathrm{Cu}$ & 3.1 & 147 & $5 \sim 8$ & 50 \\
\hline
\end{tabular}

对填充 Al-Mg 焊丝时力学性能最佳的试样进行 观察, 如图 14、表 12 所示。结果表明, 靠近钢母 材一侧为粗大的锯齿状组织, 靠近钎缝一侧为细小 的针状组织, 界面处 1 4 号检测点的元素含量与比 例表明存在 $\mathrm{Fe}-\mathrm{Al}$ 金属间化合物, 而钎缝一侧的 5 6 号检测点处, 主要是 $\alpha-\mathrm{Al}$ 固溶体, 其中 $\mathrm{Mg}$ 元素 的原子百分比达到 3.04\%。对比已有测试点中 $\mathrm{Mg}$ 元素在钎缝及界面过渡层中的含量变化, 其在钎缝 及过渡层中最大原子百分比分别为 $3.48 \% 、 0.31 \%$, 可见 $\mathrm{Mg}$ 元素并未进入 $\mathrm{Fe}-\mathrm{Al}$ 金属间化合物中, 主 要聚集在界面的钎缝一侧。当高温熔池接触钢母材 时, 大量 $\mathrm{Fe}$ 参与界面反应生成 $\mathrm{Fe}-\mathrm{Al}$ 金属间化合物, 其厚度在 $8 \sim 12 \mu \mathrm{m}$ 分布, 图 14 中标注了最大厚度, 可知 $\mathrm{Mg}$ 元素对它们的生长抑制效果欠佳, 导致钢 母材表面出现锯大量齿状组织。

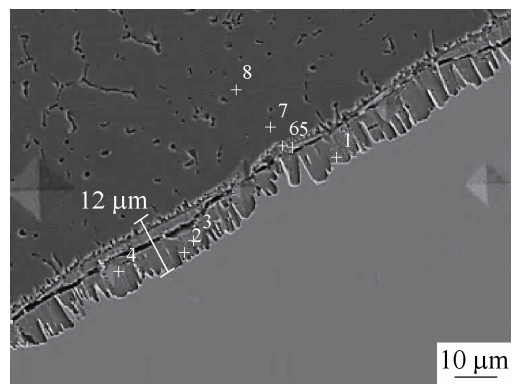

图 14 填充 Al-Mg 焊丝时钢母材侧界面
表 12 填充 Al-Mg 焊丝时钢母材侧 EDS 分析(原子分数) \%

\begin{tabular}{cccccc}
\hline 点 & $\mathrm{Al}$ & $\mathrm{Mg}$ & $\mathrm{Si}$ & $\mathrm{Cu}$ & $\mathrm{Fe}$ \\
\hline 1 & 20.89 & 0.10 & 0.34 & 0.10 & 78.57 \\
2 & 69.14 & 0.10 & 0.32 & 0.12 & 30.32 \\
3 & 74.75 & 0.31 & 0.44 & 0.05 & 24.46 \\
4 & 73.76 & 0.10 & 0.25 & 0.07 & 25.83 \\
5 & 91.36 & 3.04 & 0.10 & 0.13 & 5.37 \\
6 & 95.90 & 2.65 & 0.00 & 0.02 & 1.43 \\
7 & 96.19 & 3.21 & 0.06 & 0.09 & 0.45 \\
8 & 96.10 & 3.48 & 0.04 & 0.08 & 0.30 \\
\hline
\end{tabular}

对填充 Al-Si 焊丝时力学性能最佳的试样进行 观察, 如图 15、表 13 所示。在界面处为小锯齿状 组织, 界面处 1 5 号检测点显示此处主要是 Fe- $\mathrm{Al}$ 金属间化合物, 其中 5 号检测点 $\mathrm{Si}$ 元素原子百分比 达到 3.32\%, 而在钎缝侧的 6 号检测点, $\mathrm{Si}$ 元素原 子百分比降低至 $1.60 \%$, 可见不同于 $\mathrm{Mg}$ 元素仅在 脆性相外侧表面积聚, $\mathrm{Si}$ 元素以固溶的方式进入了 $\mathrm{Fe}-\mathrm{Al}$ 金属间化合物中, 在一定程度上抑制了脆性 相的生长。

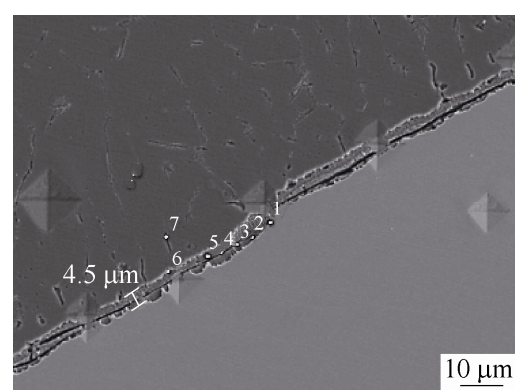

图 15 填充 Al-Si 焊丝时钢母材侧界面

表 13 填充 AI-Si 焊丝时钢母材侧 EDS 分析(原子分数) \%

\begin{tabular}{cccccc}
\hline 点 & $\mathrm{Al}$ & $\mathrm{Mg}$ & $\mathrm{Si}$ & $\mathrm{Cu}$ & $\mathrm{Fe}$ \\
\hline 1 & 25.48 & 0.10 & 1.17 & 0.11 & 73.15 \\
2 & 67.22 & 0.12 & 2.18 & 0.00 & 30.48 \\
3 & 66.45 & 0.10 & 2.04 & 0.06 & 31.34 \\
4 & 65.59 & 0.09 & 1.74 & 0.02 & 32.56 \\
5 & 70.76 & 0.18 & 3.32 & 0.16 & 25.57 \\
6 & 90.61 & 0.32 & 1.60 & 0.07 & 7.40 \\
7 & 95.94 & 0.52 & 2.16 & 0.09 & 1.30 \\
\hline
\end{tabular}

对填充 $\mathrm{Al}-\mathrm{Cu}$ 焊丝时力学性能最佳的试样进行 观察, 如图 16、表 14 所示。可见界面处有短须状 组织, 界面处 1 3 号检测点的结果显示此处主要是 固溶了部分 $\mathrm{Al}$ 元素的 $\mathrm{Fe}$ 基体, 而 4 5 号检测点发 现过渡层成分主要是 $\mathrm{Fe}-\mathrm{Al}$ 金属间化合物相, 在 6 号检测点处, 发现此处 $\mathrm{Cu}$ 元素的原子百分比高达 $11.18 \%$, 远大于 5 号检测点的 $1.62 \%$, 而 $\mathrm{Fe}$ 元素的 原子百分比仅为 $22.16 \%$, 远小于 5 号的 $48.89 \%$, 此外 6 号检测点中 $\mathrm{Al} 、 \mathrm{Fe} 、 \mathrm{Cu}$ 三种元素的原子数 目比为 $6: 2: 1$, 不符合生成 $\mathrm{Al}-\mathrm{Fe}-\mathrm{Cu}$ 三元相的条 件, 分析认为由于 $\mathrm{Cu}$ 与 $\mathrm{Fe}$ 原子有类似的晶体结构, 
导致 $\mathrm{Cu}$ 将替代部分 $\mathrm{Fe}$ 原子的位置, 导致 $\mathrm{Cu}$ 元素 骤升而 $\mathrm{Fe}$ 元素骤降, 此处的组织成分有可能是固溶 了 $\mathrm{Cu}$ 原子的 $\mathrm{FeAl}_{3}$ 相。在 7 号检测点处发现 $\mathrm{Al}$ 、 $\mathrm{Fe} 、 \mathrm{Cu}$ 三种元素的原子数目比为 $20: 2: 1$, 在过 渡层最外侧的 8 号检测点处, $\mathrm{Cu}$ 元素的原子百分 比达到 $23.05 \%$, 分析认为此处主要是 $\mathrm{Al}_{2} \mathrm{Cu}$ 共晶, 其中 $\mathrm{Cu}$ 元素的含量比 10 号钎缝内部的 $\mathrm{Al}_{2} \mathrm{Cu}$ 共 晶中的含量还高, 可见 $\mathrm{Cu}$ 元素大量聚集在界面过 渡层处。

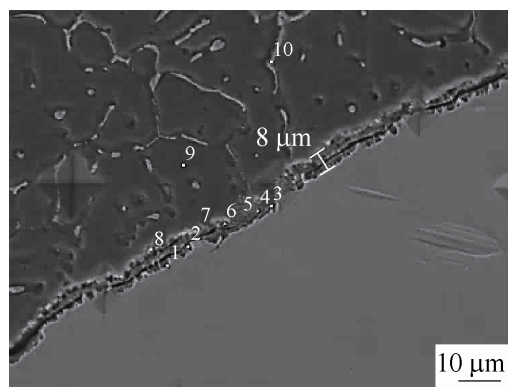

图 16 填充 $\mathrm{Al}-\mathrm{Cu}$ 焊丝时钢母材侧界面

表 14 填充 Al-Cu 焊丝时钢母材侧 EDS 分析(原子分数) \%

\begin{tabular}{cccccc}
\hline 测点 & $\mathrm{Al}$ & $\mathrm{Mg}$ & $\mathrm{Si}$ & $\mathrm{Cu}$ & $\mathrm{Fe}$ \\
\hline 1 & 4.15 & 0.04 & 0.08 & 0.65 & 95.08 \\
2 & 16.24 & 0.08 & 0.16 & 1.35 & 82.16 \\
3 & 23.72 & 0.06 & 0.16 & 0.74 & 75.32 \\
4 & 47.29 & 0.05 & 0.30 & 1.28 & 51.08 \\
5 & 49.21 & 0.10 & 0.18 & 1.62 & 48.89 \\
6 & 65.62 & 0.26 & 0.79 & 11.18 & 22.16 \\
7 & 86.49 & 0.89 & 0.35 & 4.21 & 8.06 \\
8 & 70.92 & 0.57 & 0.65 & 23.05 & 4.79 \\
9 & 99.05 & 0.42 & 0.04 & 0.36 & 0.12 \\
10 & 82.69 & 0.68 & 0.51 & 15.63 & 0.49 \\
\hline
\end{tabular}

\section{3 结论}

(1) 预涂 Nocolok 钎剂, 可改善液态钎料在钢 母材表面的润湿性, 钎剂还可起到热量壁垒、化学 壁垒的作用, 在焊接过程中, 可有效减少界面处 $\mathrm{Fe}$ 基体熔化及 $\mathrm{Fe}$ 原子向钎缝内部的扩散。

(2) 在钢母材侧预制 $60^{\circ}$ 坡口比预制 $45^{\circ}$ 坡口时 的激光熔钎焊接头力学性能更好。在钢母材侧预制 $60^{\circ}$ 坡口的基础上, 再给钢母材预制的 $\mathrm{V}$ 型槽可起 到部分 “钩爪” 效果, 但是由于界面处应力及温度 场的改变, 达不到提高接头力学性能的效果。

(3) 填充 $\mathrm{Al}-\mathrm{Cu}$ 焊丝时, 界面层厚 $5 \sim 8 \mu \mathrm{m}$, 过 渡层主要是 $\mathrm{Fe}-\mathrm{Al}$ 金属间化合物, $\mathrm{Cu}$ 元素将通过置 换 $\mathrm{Fe}$ 元素的方式进入 $\mathrm{Fe}-\mathrm{Al}$ 化合物中减小脆性, 当 激光功率为 $3.1 \mathrm{~kW}$ 时, 接头抗拉强度可达 $159 \mathrm{MPa}$ 。

(4) 填充 $\mathrm{Al}-\mathrm{Mg}$ 焊丝时, 界面层厚 $8 \sim 12 \mu \mathrm{m}$,
过渡层主要是 $\mathrm{Fe}-\mathrm{Al}$ 金属间化合物, $\mathrm{Mg}$ 元素将聚集 在脆性相外侧, 当激光功率为 $3 \mathrm{~kW}$ 时, 接头抗拉 强度可达 $118 \mathrm{MPa}$ 。

(5) 填充 Al-Si 焊丝时，界面层厚 3 4.5 $\mu \mathrm{m}$, 过渡层主要是 $\mathrm{Fe}-\mathrm{Al}$ 金属间化合物, $\mathrm{Si}$ 元素将固溶 在 $\mathrm{Al}-\mathrm{Fe}$ 二元相中抑制其生长, 当激光功率为 $3 \mathrm{~kW}$ 时, 接头抗拉强度可达到 $129 \mathrm{MPa}$ 。

\section{参 考 文 献}

[1] 张希良. 中国新能源汽车产业发展现状与展望[J]. 环 境保护，2013，41(10): 24-27.

ZHANG Xiliang. Development status and prospect of china's new energy automobile industry[J]. Environmental Protection, 2013, 41(10): 24-27.

[2] 王海良, 秦振华. 乘用车燃料消耗量标准推进中国节能 与新能源汽车的发展 [J]. 交通节能与环保, 2017, 13(1): 17-22.

WANG Hailiang, QIN Zhenhua. Fuel consumption standard of passenger car promotes the development of energy saving and new energy vehicles in China[J]. Traffic Energy Saving \& Environmental Protection, 2017, 13(1): 17-22.

[3] 李永兵, 马运五, 楼铭, 等. 轻量化多材料汽车车身连 接技术进展[J]. 机械工程学报, 2016，52(24)：1-23.

LI Yongbing, MA Yunwu, LOU Ming, et al. The development of lightweight multi material automobile body joining technology $[\mathrm{J}]$. Journal of Mechanical Engineering, 2016, 52(24): 1-23.

[4] LI X, MA H, SHEN Z. Research on explosive welding of aluminum alloy to steel with dovetail grooves[J]. Materials \& Design, 2015, 87: 815-824.

[5] MACWAN A, KUMAR A, CHEN D L. Ultrasonic spot welded 6111-T4 aluminum alloy to galvanized high-strength low-alloy steel : Microstructure and mechanical properties[J]. Materials \& Design, 2017, 113: 284-296.

[6] LEITAO C, ARRUTI E, ALDANONDO E, et al. Aluminium-steel lap joining by multipass friction stir welding[J]. Materials \& Design, 2016, 106: 153-160.

[7] LI C L, FAN D, WANG B. Characteristics of TIG arc-assisted laser welding-brazing joint of aluminum to galvanized steel with preset filler powder[J]. Rare Metals, 2015, 34(9): 650-656.

[8] 王晓虹, 谷晓燕, 孙大千. 钢/铝异种金属激光焊接头 界面特性的研究[J]. 机械工程学报, 2017(4)：26-33.

WANG Xiaohong, GU Xiaoyan, SUN Daqian. Study on the interface characteristics of laser welded joint of steel/aluminum dissimilar metals[J]. Journal of 
Mechanical Engineering, 2017(4): 26-33.

[9] ZHANG G F, SU W, ZHANG J X, et al. Friction stir brazing: A novel process for fabricating $\mathrm{Al} /$ Steel layered composite and for dissimilar joining of Al to steel[J]. Metallurgical and Materials Transactions A, 2011, 42(9): 2850-2861.

[10] 张启运, 庄鸿寿. 钎焊手册 [M]. 北京: 机械工业出版 社, 2008.

ZHANG Qiyun , ZHUANG Hongshou. Brazing manual[M]. Beijing: China Machine Press, 2008.

[11] FIELD D J, STEWARD N I. Mechanical aspect of the nocolok flux brazing process[R]. SAE, 870186, 1987.

[12] 章桥新. 论金属间化合物的脆性及其克服途径 [J]. 稀 有金属材料与工程, 1990(6): 27-31.

ZHANG Qiaoxin. On the brittleness of intermetallic compounds and the way to overcome it[J]. Rare Metal Materials and Engineering, 1990(6): 27-31.

[13] MASSALSKI T B, OKAMOTO H, SUBRAMANIAN P $\mathrm{R}$, et al. Binary alloy phase diagrams[M]. Materials Park, Ohio: ASM International, 1990.

[14] HEUMANN T, DITTRICH N. Structure character of the $\mathrm{Fe}_{2} \mathrm{Al}_{5}$ intermetallics compound in hot dip aluminizing process[J]. Zeitschrift fur Metallkunde, 1959, 50(10): 617-625.

[15] EGGELER G, VOGEL H, FRIEDRICH J, et al. Targer preparation for transmission electron microscopic identification of FeAl3 ( $\theta$-Phase) in hot-dip Aluminized low-alloy steel[J]. Practical Metallography, 1985, 22(4): 163-170

[16] EGGELER G, AUER W, KAESCHE H. Reactions between low alloyed steel and initially pure as well as iron saturated aluminum melts between 670 and $800{ }^{\circ} \mathrm{C}[\mathrm{J}]$. Zeitschrift fur Metallkunde, 1986, 77(4): 239-244.

[17] SONG J L, LIN S B, YANG C L, et al. Effects of Si additions on intermetallic compound layer of aluminumsteel TIG welding-brazing joint[J]. Journal of Alloys \& Compounds, 2009, 488(1): 217-222.

[18] LEMMENS B, CORLU B, DE S J, et al. The effect of Si on the intermetallic formation during hot dip aluminizing[J]. Advanced Materials Research, 2014, 922: 429-434.

[19] NAZARI K A, SHABESTARI S G. Effect of micro alloying elements on the interfacial reactions between molten aluminum alloy and tool steel[J]. Journal of Alloys \& Compounds, 2009, 478(1-2): 523-530.

作者简介: 张建勋(通信作者), 男, 1958 年出生, 博士, 教授, 博士研 究生导师。主要研究方向为先进材料接合与连接、智能焊接与再制造、 焊接结构服役安全评价。

E-mail: jxzhang@mail.xjtu.edu.cn

江旭, 男, 1993 年出生。主要研究方向为激光焊接技术。

E-mail: jiangxujx@stu.xjtu.edu.cn 